\title{
Enucleation of a Rare Hybrid Odontogenic Lesion in the Anterior Maxilla of a 10-Year-Old Boy
}

\author{
Amanda Gruza, DMD ${ }^{1,2}$, Jason Choi, DDS, $F R C D(C)^{1}$, Curtis Chen, DDS, PhD, Diplomat ABOMR and \\ Catherine F. Poh, DDS, PhD, FRCD(C) and Diplomat ABOMP ${ }^{1^{*}}$
}

${ }^{1}$ Faculty of Dentistry, University of British Columbia, Vancouver, BC, Canada

${ }^{2}$ College of Dentistry, University of Saskatchewan, Saskatoon, SK, Canada

${ }^{3}$ Faculty of Dentistry, University of Washington, Seattle, WA, USA

\begin{abstract}
Hybrid odontogenic lesions are a well-recognized phenomenon. They most often show features of various odontogenic neoplasms arising within cyst-like structures. The etiopathogenesis of hybrid odontogenic lesions is not well defined, however, the presence of numerous, closely approximated sources of pluripotent epithelial cells in the developing jaws is likely associated with their development. This case highlights an interesting mixed radiolucent and radiopaque, expansile cystic lesion with a follicular relationship to an unerupted tooth in a young child. Histologically, the lesion showed a combination of dentigerous cyst, calcifying odontogenic cyst and adenomatoid odontogenic tumor. Close radiographic proximity of the hybrid odontogenic lesion to a focal expansion of the gubernacular canal suggests that the adenomatoid odontogenic tumor portion of the hybrid lesion may have arisen directly from this structure, resulting in delayed eruption of the adjacent tooth and subsequent development of a dentigerous cyst with foci of calcifying odontogenic cyst. Complete enucleation of the cystic structure with retention of the associated tooth was performed, with a 6-month follow-up showing complete healing of the surgical site and no evidence of recurrence of the cystic lesion. To date, there have been only six reported cases of lesions with features of both calcifying odontogenic cyst and adenomatoid odontogenic tumor. The current case represents the first documented case of a dentigerous cyst with foci of both calcifying odontogenic cyst and adenomatoid odontogenic tumor. Given the variable nature and biological behavior of the odontogenic entities that compose hybrid odontogenic lesions, unusual clinical presentations and unexpected progression are possible. Further documentation of such hybrid lesions is necessary to fully elucidate how these entities behave clinically, radiographically and pathologically.
\end{abstract}

Keywords

Hybrid odontogenic tumor, Hybrid odontogenic cyst, Hybrid odontogenic neoplasm, Dentigerous cyst, Calcifying odontogenic cyst, Adenomatoid odontogenic cyst

\section{Introduction}

Recently, there have been several publications of socalled "hybrid odontogenic tumors" [1-14] including those with features of various odontogenic neoplasms arising within cyst-like structures [9-14]. The hybrid odontogenic lesions arise most commonly in the first two decades of life and appear to affect males and females equally [1-20]. The etiopathogenesis of these hybrid odontogenic lesions has not been fully elucidated. Odontogenic cysts and tumors are known to arise from the various sources of pluripotent epithelial cells within the oral cavity, including the dental lamina, gubernacular cord, odontogenic epithelial rests and reduced enamel epithelium [21-23]. In the early mixed dentition, developing tooth buds are often in close proximity to many of these sources of pluripotent epithelial cells. It is thus conceivable, that the tooth buds and early odontogenic cysts and tumors arising from these sources could influence one another in their respective development. It is also possible, given their close proximity, that these structures could eventually merge resulting in the formation of hybrid odontogenic lesions with diverse histological features. This case report presents radiographic and histologic evidence

*Corresponding author: Dr. Catherine F. Poh, Faculty of Dentistry, University of British Columbia, 2199 Wesbrook Mall, Vancouver, BC, V6T1Z3, Canada, Tel: 604-675-8000 ext 7072, Fax: 604-675-8263

Accepted: April 18, 2019

Published online: April 20, 2019

Citation: Gruza A, Choi J, Chen C, et al. (2019) Enucleation of a Rare Hybrid Odontogenic Lesion in the Anterior Maxilla of a 10-Year-Old Boy. Archives Oral Maxillofac Surg 2(1):18-24 


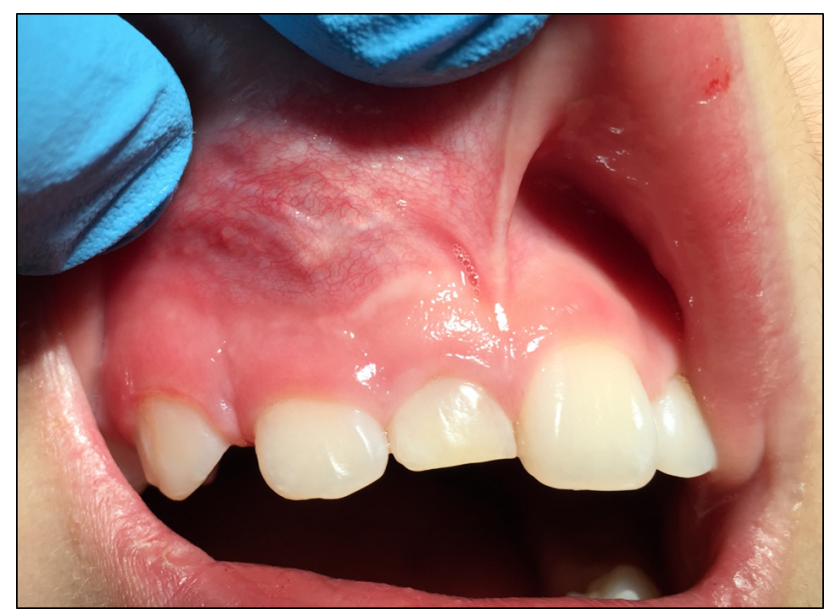

Figure 1: Clinical photo showing an expansile lesion in the right anterior maxilla extending from the mesial aspect of the right canine to the mesial aspect of the left central incisor with associated buccal expansion.

supportive of this theory. We present a cyst-like lesion with a follicular relationship to an unerupted tooth and close proximity to a gubernacular cord, histologically consistent with a hybrid odontogenic lesion composed of a dentigerous cyst (DC) with foci of calcifying odontogenic cyst (COC) and adenomatoid odontogenic tumor (AOT).

\section{Case Report}

A 10-year-old otherwise healthy Asian boy presented to his family dentist with delayed eruption of his right permanent maxillary central incisor. Upon clinical examination, a large, asymptomatic swelling was observed in the right anterior maxilla. The patient was subsequently referred to an oral and maxillofacial surgeon for further evaluation. Extra-oral examination showed age appropriate development with no gross facial asymmetry, no submandibular or cervical lymphadenopathy, and no cranial nerve deficits. Intra-oral examination revealed a swelling in the right anterior maxilla adjacent the roots of the right central and lateral incisors (Figure 1). The lesion was non-mobile and non-tender on palpation. Buccal bony expansion was apparent, without evidence of palatal expansion. The overlying mucosa was intact and showed an area of faint blue discoloration. There was no associated paresthesia, nor anesthesia. Dental development was within normal limits, with the exception of an unerupted permanent maxillary central incisor and associated retained primary maxillary central incisor. All erupted teeth were noted to be periodontally sound and non-carious. There was no mobility of the teeth adjacent the lesion, and vitality tests were within normal limits.

Panoramic imaging showed a well-defined, unilocular, cystic radiolucency in the right anterior maxilla associated with a superiorly displaced permanent right central incisor and distally displaced root of the permanent right lateral incisor. Conebeam computed tomography (CBCT) confirmed the presence of a well-defined, unilocular, cystic, hypodense lesion associated with the impacted right central incisor within the anterior maxilla. The cystic lesion extended
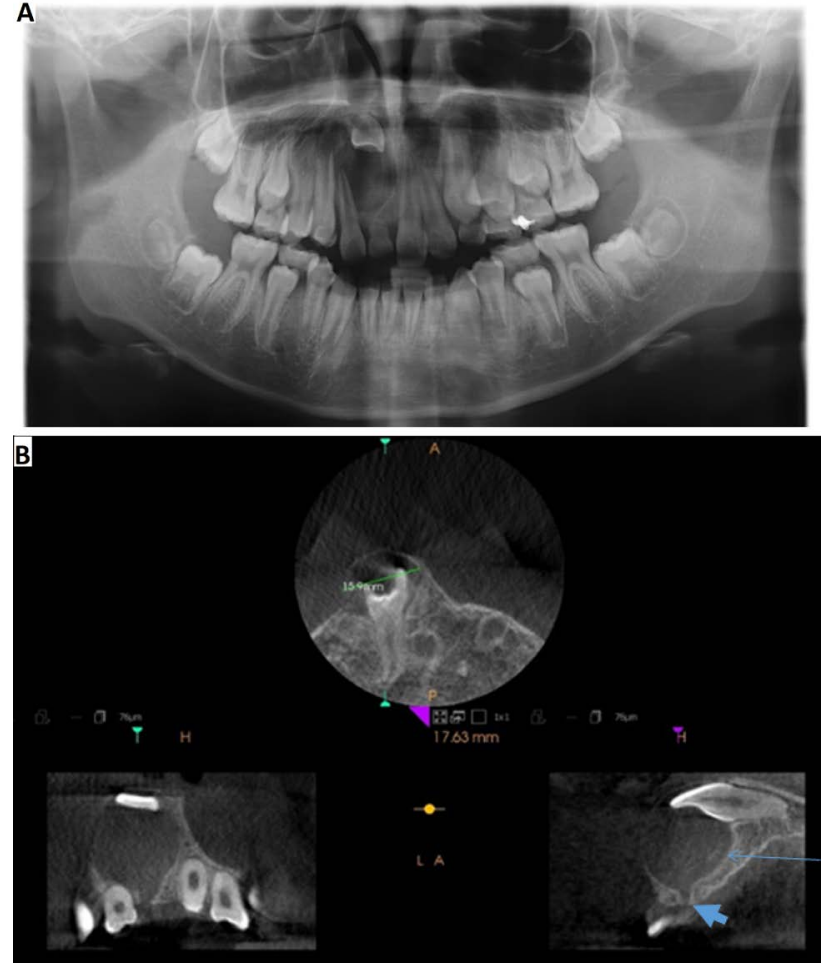

Figure 2: A) Pre-operative panoramic image showing a cystic structure associated with the crown of an impacted right central incisor; B) Pre-operative cone beam computed tomography showing a hypodense cystic structure associated with the crown of an impacted right central incisor and corresponding gubernacular canal (thick blue arrowhead); faint hyperdense entities are present in the posterior aspect of the cystic lumen (thin blue arrow).

from the maxillary right canine to the left central incisor. Hyperdense entities were noted within the cystic lumen (thin blue arrow, Figure 2). The lesion appeared attached to the unerupted central incisor at the cementoenamel junction (CEJ), with displacement of the tooth superiorly where it approximated the nasal floor. A gubernacular canal was noted to extend inferiorly from the cystic lesion through the alveolar bone (thick blue arrowhead, Figure 2).

Informed consent was obtained from the patient and his parents, and surgical excision of the lesion was completed under general anesthesia. A full thickness flap was elevated and the lesion was aspirated with an 18-gauge needle on a $10 \mathrm{cc}$ syringe. The aspirate revealed a yellow fluid mixed with blood. The cyst-like lesion was detached from the CEJ of the unerupted maxillary right central incisor, fully enucleated and placed in $10 \%$ buffered formalin for histopathologic examination. The impacted right central incisor was left in place to allow for subsequent unimpeded eruption. The site of enucleation was debrided, irrigated and closed with 3-0 gut sutures.

Gross examination of the specimen showed a $1.8 \times 1.6 \times$ $1.2 \mathrm{~cm}$ ovoid dark grey-brown, collapsed cystic structure. The specimen was serially sectioned and stained with hematoxylin and eosin. Microscopic examination showed a cystic cavity lined, almost entirely, by thin, non-keratinized stratified 


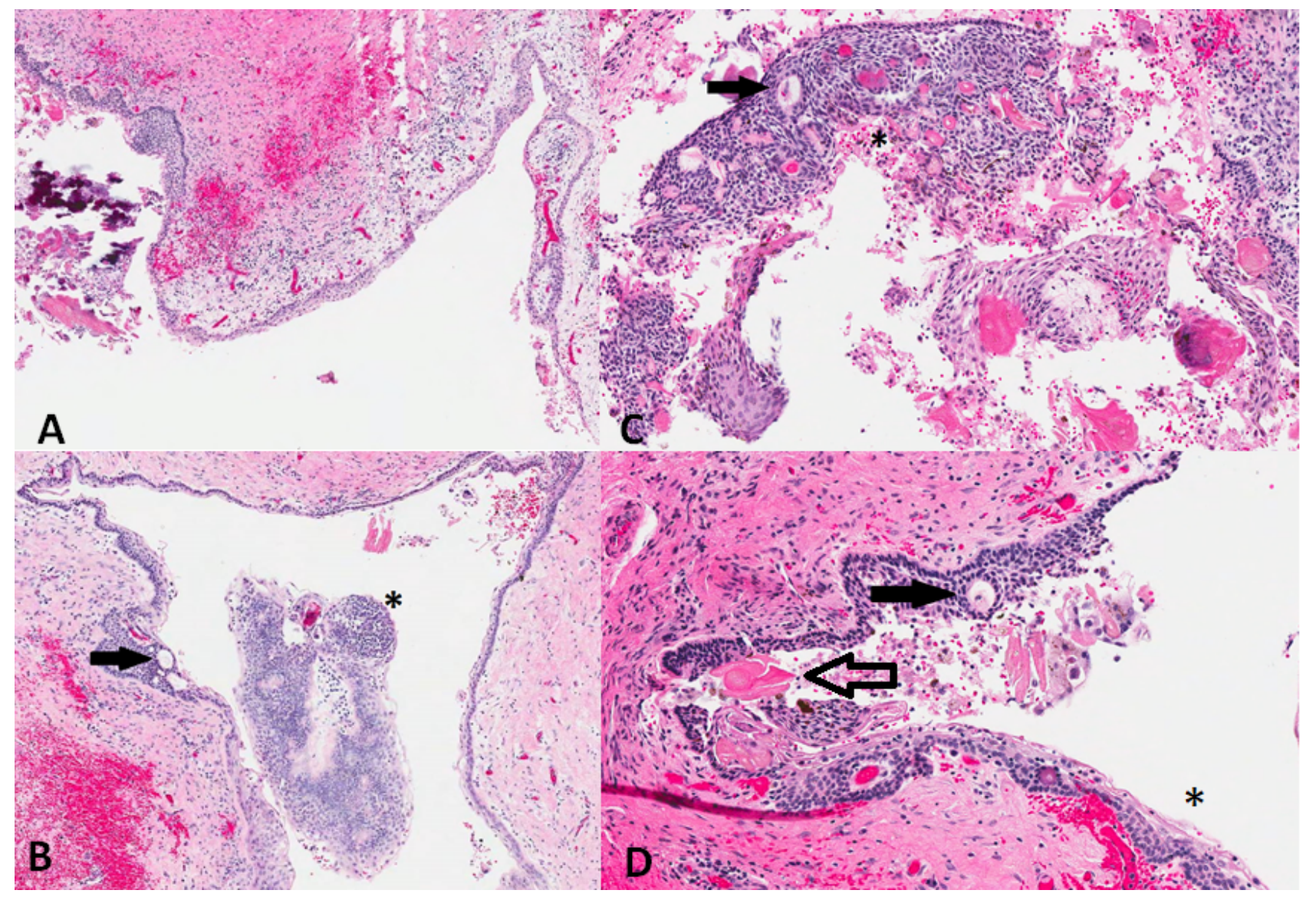

Figure 3: Photomicrograph of the cyst. A) Transition of cystic lining epithelium from dentigerous cyst (DC, right) to calcifying odontogenic cyst (COC, left); B) Transition of cystic lining epithelium from DC (right) to adenomatoid odontogenic tumor (AOT, left and center). The AOT component shows duct-like spaces (black arrow) and epithelial whorls (asterisk); C) Transition from COC (right) to AOT (left). The AOT component shows duct-like spaces (black arrow). The COC component shows eosinophilic ghost cells (asterisk); (D) Transition from AOT (black arrow identifying duct-like structures of the AOT component), COC (open arrow identifying collections of ghost cells in the COC component) to DC (asterisk identifying transition to epithelium of DC). (Hematoxylin and Eosin staining; original magnification, $A \& B, 100 X ; C \& D, 200 X)$

squamous epithelium, consistent with that of a conventional DC (Figure 3A). Contiguous with the DC-type lining were areas showing palisaded columnar basal cells, areas of loosely and densely arranged stellate reticular cells, and collections of eosinophilic ghost cells with calcification, consistent with the features of a COC (Figure 3C and Figure 3D). Additional foci showed nodular thickening of the epithelium with formation of whorls and ductal structures consistent with an AOT (Figure 3B, Figure 3C and Figure 3D). The final histopathologic diagnosis provided was that of a hybrid odontogenic lesion consisting of a COC and AOT arising from a DC.

The patient's recovery was unremarkable. Follow up evaluations took place one week post-operatively and 6-months post-operatively. Follow up radiographic imaging, completed at the 6-month post-operative evaluation, confirmed complete healing of the surgical site with no recurrence of the cystic lesion. The right central incisor remained impacted and the gubernacular canal remained intact (thick blue arrowhead, Figure 4B). Subsequent surgical exposure and orthodontic traction of the permanent right central incisor were performed (Figure 4A). Annual follow-ups have been scheduled to ensure a lack of recurrence of the maxillary lesion.

\section{Discussion}

Based on the clinical examination and radiographic presence of a mixed hypo- and hyper-dense lesion with a follicular relationship to an impacted tooth, the clinical differential diagnosis included conventional dentigerous cyst (DC) with dystrophic calcification, adenomatoid odontogenic tumor (AOT), calcifying odontogenic cyst (COC), and calcifying epithelial odontogenic tumor (CEOT) [24].

The DC is a developmental odontogenic cyst which originates from a separation of the follicle from an unerupted tooth with subsequent accumulation of fluid between the odontogenic epithelium and the crown. It is the most common odontogenic cyst. The most commonly associated impacted tooth is the mandibular third molar. Maxillary third molars, maxillary canines, and mandibular second premolars are also commonly associated with DCs. Most of these cysts remain asymptomatic, showing symptoms only in the presence of prominent bony expansion or secondary infection. Radiographically, the DC presents as a unilocular radiolucency attached to the unerupted tooth at the CEJ. The border most often appears corticated and well-defined. 


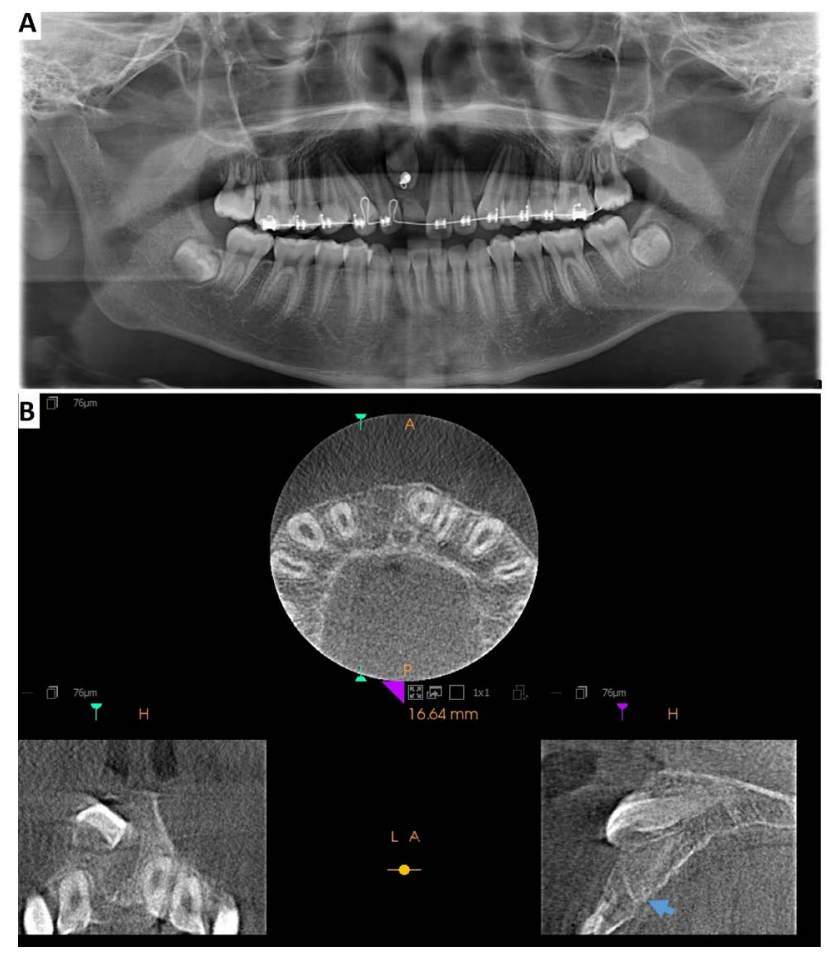

Figure 4: A) Six-month post-operative panoramic image (taken just after surgical exposure and prior to the initiation of orthodontic traction of the right central incisor) showing a healed surgical site with no recurrence of the cystic lesion; B) Six-month post-operative CBCT showing a healed surgical site in the anterior maxilla, unerupted right central incisor and intact gubernacular canal (thick blue arrowhead).

Displacement of the associated unerupted tooth can occur, as can resorption of adjacent roots. Dystrophic calcification within the cyst wall can result in a mixed radiolucent and radio-opaque appearance radiographically, mimicking other odontogenic tumors [25]. Histologically, non-inflamed DCs show a thin, attenuated squamous epithelial lining with absence of keratin and rete ridge formation. Dystrophic calcification may be identified in the epithelial cyst lining.

AOTs are benign neoplasms thought to arise from odontogenic epithelium, odontogenic rests, or the gubernacular cord. The vast majority of these tumors occur in adolescence, namely the second decade of life. The AOT is found primarily in the anterior maxilla, associated with an unerupted canine. A female predilection is noted. Most AOTs are asymptomatic and are discovered following investigation of an unerupted permanent tooth. Rare cases have been associated with enlargement of the adjacent bone. Radiographically, the AOT presents as a unilocular radiolucency with small radio-opacities, representing internal calcifications, surrounding the crown of an unerupted tooth. The border is most often corticated and well-defined. Compared to the DC, the cystic attachment point of an AOT is often located along the root, apical to the CEJ. The AOT, which has been documented to occur in combination with COCs $[16,17,19-21,26]$, CEOTs [21] and odontomas [21], is known to present with a variety of histoarchitectural patterns characteristically showing formation of duct-like or rosette-like structures [21,27].
COCs have been categorized as cysts, neoplasms, and hamartomas [28]. This uncertainty results from the numerous clinical, histopathological, and behavioural variations this lesion may present with. Clinically, the most common presentation is that of an asymptomatic bony expansion in the anterior maxilla or mandible. Radiographically, COCs appear most often as unilocular radiolucencies, although multi-locularity can be seen. The border is well-defined and in one third of cases encompasses an unerupted tooth. The most commonly associated tooth is the maxillary or mandibular canine. COCs are often associated with radiopacities within the cystic structure. Displacement or resorption of adjacent roots is often present. Since it was first described [29], the COC has been reported to have substantial histopathologic variability. Reports of cystic, solid, and hybrid variants have been described extensively in the published literature [15,28,30,31]. The most common presentation is that of a benign cystic neoplasm, characterized by an ameloblastoma-like epithelium with ghost cells which may calcify [32]. In addition to the variable histologic presentations, calcifying odontogenic cysts also occur in conjunction with other known odontogenic tumors such as ameloblastoma [33], ameloblastic fibroma [33], previously classified ameloblastic fibro-odontoma [31,33], odontoma [15,29,34], and AOT [15-20]. These rare hybrid odontogenic neoplasms appear to retain separate and distinct histopathological features while existing within a single neoplastic entity.

The present case highlights an interesting mixed radiolucent and radiopaque, expansile cystic lesion with a follicular relationship to an unerupted tooth in a young child which histologically showed a combination of DC, COC, and AOT. To date, there have been only six reported cases of lesions with features of both COC and AOT [15-20] published in the English language literature, all reviewed in detail by Soares, et al. [19] and summarized in Table 1. The current case represents the first documented case of a DC with foci of both $\mathrm{COC}$ and AOT.

It has been suggested that the gubernacular cord, a remnant of the dental lamina [30], is the site of origin of AOTs $[35,36]$. It is plausible, that the small focus of AOT found in the present case arose directly from the gubernacular cord, suggested radiographically, by the focal expansion of the gubernacular canal (thick blue arrowhead, Figure 2).

According to the intra-follicular theory of DC formation, an erupting tooth exerts pressure on the dental follicle leading to obstruction of venous outflow [37]. This results in serum transudation, increased hydrostatic pressure, and separation of the follicle from the crown. It is thus conceivable, that in the current case, pressure was exerted on the dental follicle by both the erupting tooth and the obstruent AOT, resulting in the development of a DC. Hong, et al. described a similar process for the development of $\mathrm{COC}$ as a result of a proximate odontoma [28], suggesting that the foci of COC present in the current case may have resulted through the same process responsible for formation of the DC. The concurrent existence, and abrupt transition between a COC within a DC has also previously been described by Praetorious, et al. [31]. 


\begin{tabular}{|c|c|c|c|c|c|c|c|}
\hline & 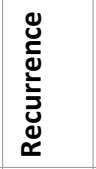 & 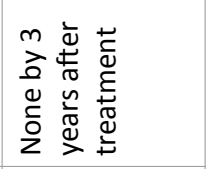 & 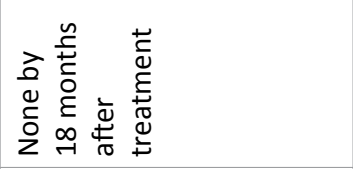 & $\frac{\pi}{z}$ & $\frac{\pi}{z}$ & 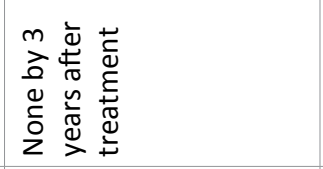 & 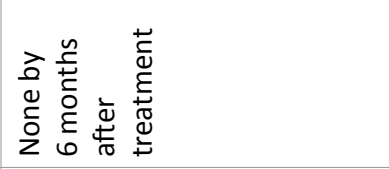 \\
\hline & 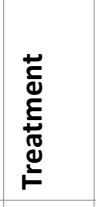 & 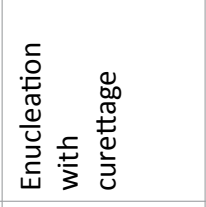 & 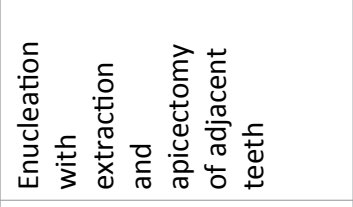 & 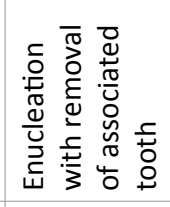 & $\frac{\pi}{z}$ & 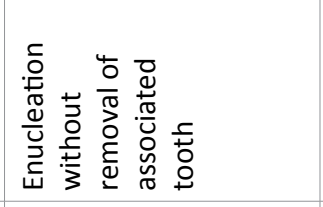 & 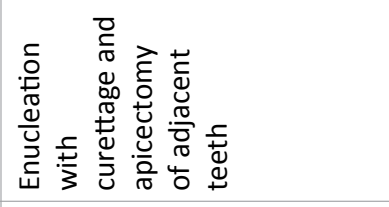 \\
\hline & 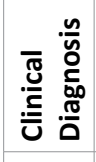 & $\frac{\pi}{z}$ & రั & $\frac{\pi}{z}$ & $\frac{\pi}{z}$ & 虽 & 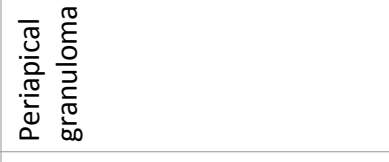 \\
\hline & 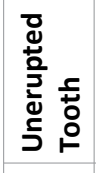 & $\frac{1}{2}$ & $\begin{array}{l}0 \\
\tilde{\Sigma} \\
z\end{array}$ & F & $\begin{array}{l}0 \\
\check{0} \\
z\end{array}$ & $\vec{F}$ & $\begin{array}{l}0 \\
\stackrel{0}{0} \\
z\end{array}$ \\
\hline & $\stackrel{\stackrel{N}{\tilde{E}} \bar{E}}{\bar{E}}$ & $\frac{\pi}{z}$ & 우 & 울 & $\stackrel{\circ}{+}$ & $\stackrel{\circ}{\dot{m}}$ & $\hat{i}$ \\
\hline & 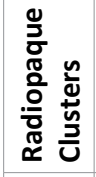 & 울 & $\stackrel{\tilde{u}}{\nu}$ & 2) & 2 & $\stackrel{y}{\nu}$ & $\frac{\pi}{z}$ \\
\hline & 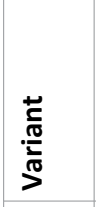 & 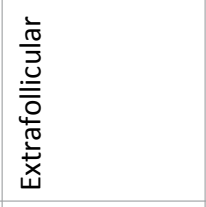 & 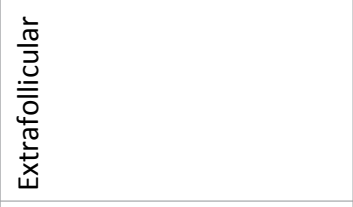 & 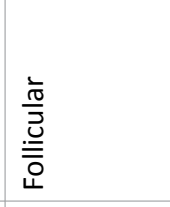 & $\frac{\pi}{z}$ & 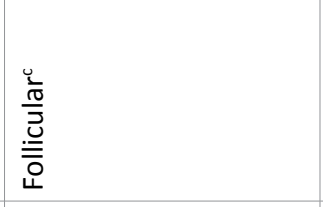 & 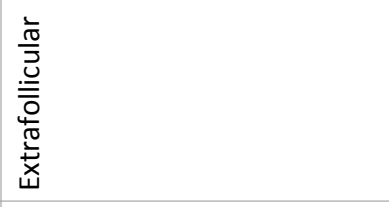 \\
\hline 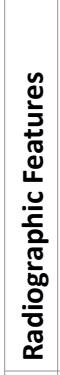 & 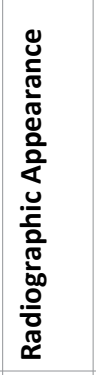 & 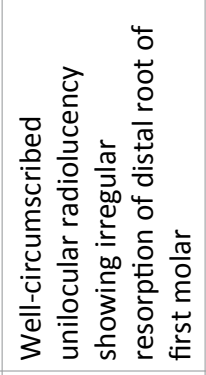 & 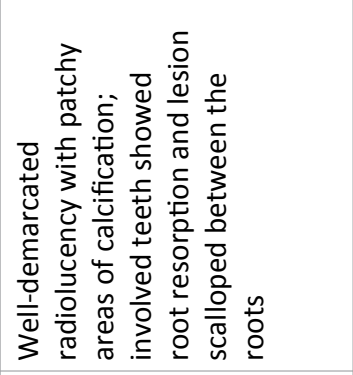 & 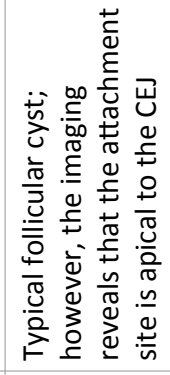 & 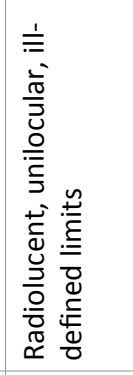 & 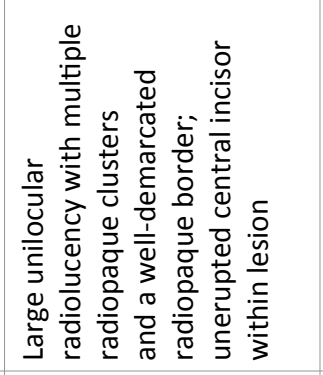 & 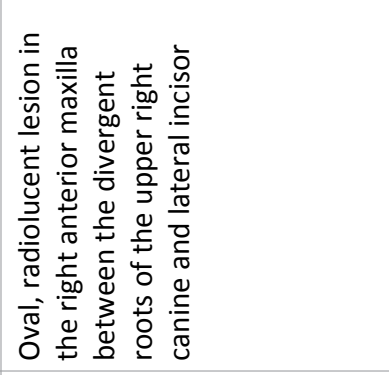 \\
\hline & 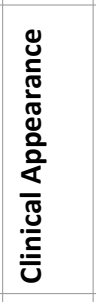 & 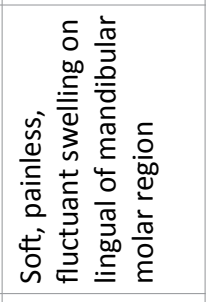 & 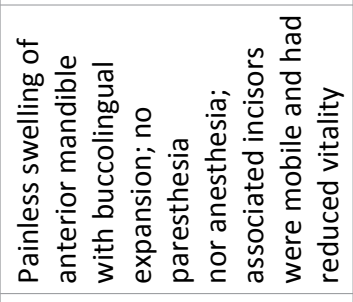 & 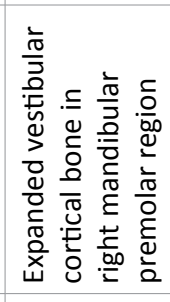 & $\frac{\pi}{2}$ & 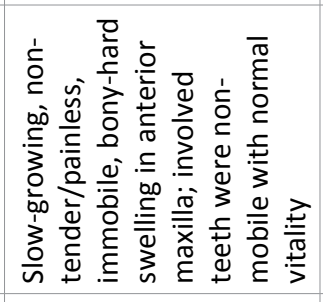 & 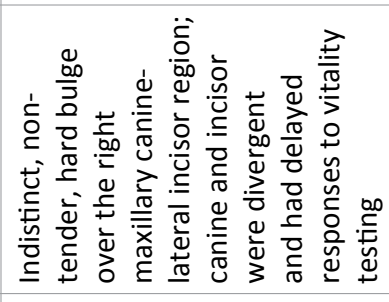 \\
\hline & $\stackrel{\Xi}{5}$ & 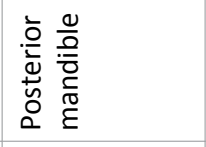 & 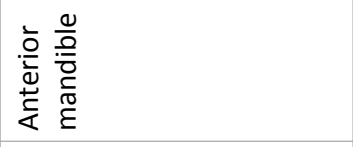 & 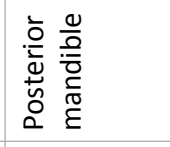 & 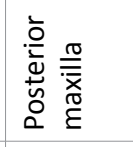 & 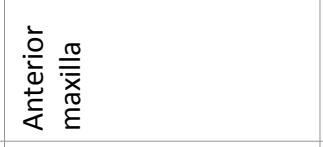 & 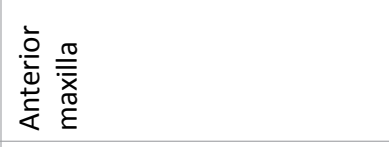 \\
\hline & 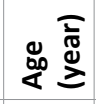 & 늠 & $\stackrel{\llcorner}{m}$ & $\vec{F}$ & 9 & $N$ & भ̧ \\
\hline & હ & 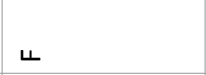 & $\Sigma$ & 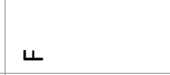 & $\Sigma$ & 4 & $\Sigma$ \\
\hline & 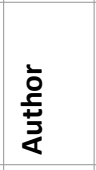 & 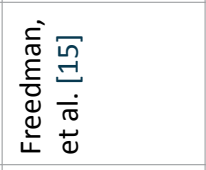 & 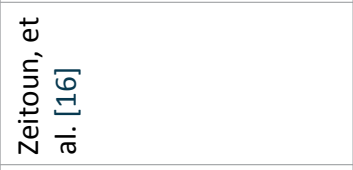 & 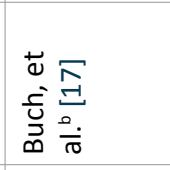 & 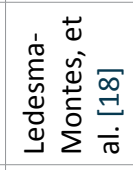 & 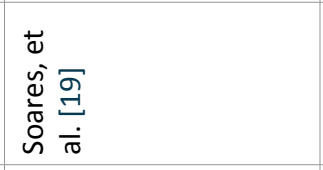 & 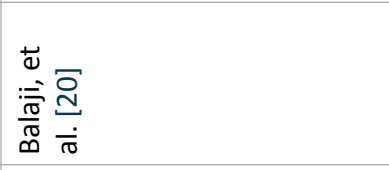 \\
\hline & 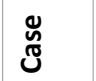 & -1 & $\sim$ & $m$ & $\sigma$ & in & 0 \\
\hline
\end{tabular}




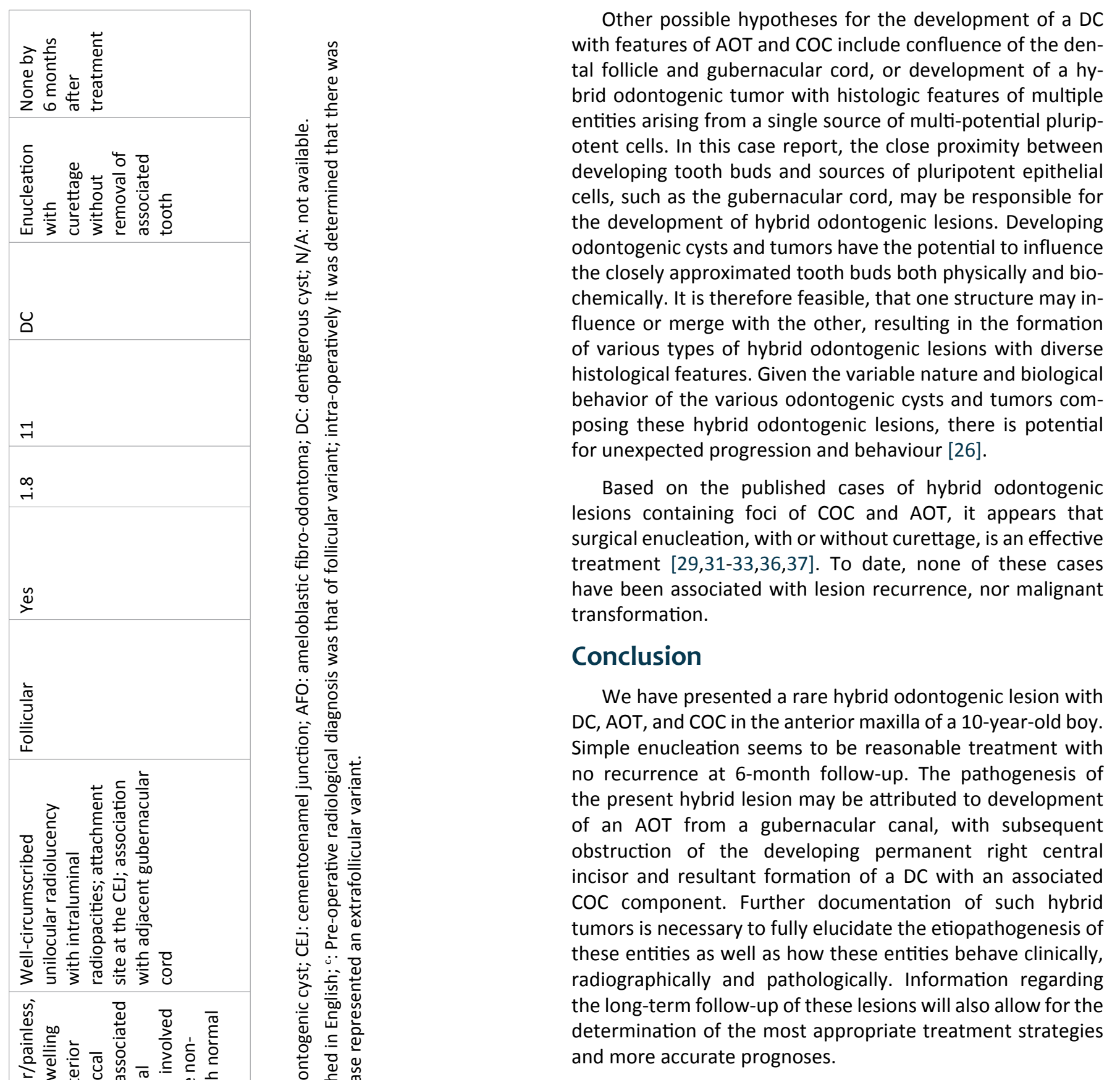

\section{Conflict of Interest Statement}

Amanda Gruza, Jason Choi, Curtis Chen and Catherine Poh declare that they have no conflicts of interest.

\section{Ethical Approval}

This article does not contain any studies with human or animal subjects performed by any of the authors. The review and report of radiographic and pathologic information is under the institutional ethical approval (UBC/BCCA H16-00611). All reporting procedures performed were in accordance with the ethical standards of the 1964 Helsinki declaration and its later amendments.

\section{References}

1. Imani R, Jamaatloo N, Seyedmajidi M, et al. (2017) Hybrid odontogenic lesion: A rare entity. Journal of Dental Materials and Techniques 6: 40-43. 
Citation: Gruza A, Choi J, Chen C, et al. (2019) Enucleation of a Rare Hybrid Odontogenic Lesion in the Anterior Maxilla of a 10-Year-Old Boy. Archives Oral Maxillofac Surg 2(1):18-24

2. Tomar U, Bishen KA, Singh A, et al. (2018) Review on pathogenesis of hybrid variant of AOT and report of a rare case. Journal of Advanced Medical and Dental Sciences Research 6: 38-42.

3. Ricardo JH, Lomonaco SH, Martínez AM (2018) Hybrid lesion formed by a complex odontoma and dentigerous cyst: Radiographic and histomorphological findings. A case report. Journal of Oral Research 7: 145-149.

4. Aristizabal Arboleda P, Sánchez-Romero C, de Almeida OP, et al. (2018) Calcifying odontogenic cyst associated with dentigerous cyst in a 15-year-old girl. International Journal of Surgical Pathology 26: 758-765.

5. Muddana K, Maloth AK, Dorankula SP, et al. (2019) Calcifying cystic odontogenic tumor associated with ameloblastoma - A rare histological variant. Indian J Dent Res 30: 144-148.

6. Gamoh S, Akiyama H, Furukawa C, et al. (2017) Calcifying cystic odontogenic tumor accompanied by a dentigerous cyst: A case report. Oncology letters 14: 5785-5790.

7. Sathyanarayana VK, Srigiri $\mathrm{H}$, Cheemalavagupalli M, et al. (2017) $A$ rare case of adenomatoid odontogenic tumour with unicystic ameloblastoma. J Clin Diagn Res 11: ZJ05- ZJ06.

8. Sreenivas SD, Lalita CS, Harsha G, et al. (2015) Multiple pathology in a single lesion: AOT associated with dentigerous cyst. Maxillofac Oral Surg 14: 215-221.

9. Majumdar S, Uppala D, Rao AK, et al. (2015) Dentigerous cyst associated with adenomatoid odontogenic tumour. J Clin Diagn Res 9: ZD01-ZD04.

10. Wadhwan V, Sharma P, Bansal V (2015) A rare case of hybrid odontogenic tumor: Calcifying epithelial odontogenic tumor combined with ameloblastoma. J Oral Maxillofac Pathol 19: 268.

11. Neuman AN, Montague L, Cohen D, et al. (2015) Report of two cases of combined odontogenic tumors: ameloblastoma with odontogenic keratocyst and ameloblastic fibroma with calcifying odontogenic cyst. Head Neck Pathol 9: 417-420.

12. Mota MR, Alves AP, Costa FW, et al. (2015) Clinicopathologic, cone-beam computed tomographic, and surgical findings in a unique maxillary hybrid odontogenic tumor. J Craniofac Surg 26: e551-e552.

13. Yamazaki M, Maruyama S, Abé T, et al. (2014) Hybrid ameloblastoma and adenomatoid odontogenic tumor: report of a case and review of hybrid variations in the literature. Oral Surg Oral Med Oral Pathol Oral Radiol 118: e12-e18.

14. Chaubey SS, Mishra SS, Degwekar SS, et al. (2013) A rare presentation of hybrid odontogenic tumor involving calcifying cystic odontogenic tumor and plexiform ameloblastoma. Contemp Clin Dent 4: 406-408.

15. Freedman PD, Lumerman H, Gee JK (1975) Calcifying odontogenic cyst: A review and analysis of seventy cases. Oral Surg 40: $93-$ 106.

16. Zeitoun IM, Dhanrajani PJ, Mosadomi HA (1996) Adenomatoid odontogenic tumor arising in a calcifying odontogenic cyst. J Oral Maxillofac Surg 54: 634-637.

17. Buch RSR, Coerdt W, Wahlmann U (2003) Adenomatoid odontogenic tumor in a calcifying odontogenic cyst. Mund Kiefer Gesichts Chir 7: 301-305.

18. Ledesma-Montes C, Gorlin RJ, Shear M, et al. (2008) International collaborative study on ghost cell odontogenic tumours: Calcifying cystic odontogenic tumour, dentinogenic ghost cell tumor and ghost cell odontogenic carcinoma. J Oral Pathol 37: 302-308.
19. Soares ECS, Costa FWG, Neto ICP, et al. (2011) Rare hybrid odontogenic tumor in a 2-year-old child. J Craniofac Surg 22: 554-558.

20. Balaji SM, Rooban T (2012) Calcifying odontogenic cyst with atypical features. Ann Maxillofac Surg 2: 82-85.

21. Neville B (2016) Oral and Maxillofacial Pathology. (4 $4^{\text {th }}$ edn), Elsevier, St. Louis, 632.

22. Woo S (2012) Oral Pathology: A Comprehensive Atlas and Text. ( $1^{\text {st }}$ edn), Elsevier/Saunders, Philadelphia, 320,340.

23. Padma Priya S, Higuchi A, Abu Fanas S, et al. (2015) Odontogenic epithelial stem cells: hidden sources. Lab Invest 95: 1344-1352.

24. Wright JM, Devilliers P (2017) Calcifying epithelial odontogenic tumour. In: El-Nagar AK, Chan JKC, Grandis JR, et al. World Health Organization Classification of Head and Neck Tumours. International Agency for Research on Cancer, Volume 9, Lyon, France, 220-221.

25. Shimizu M, Ogawa D, Okamura K, et al. (2015) Dentigerous cysts with calcification mimicking odontogenic tumors: Differential diagnosis by CT. Oral Radiology 31: 14-22.

26. Phillips MD, Closmann JJ, Baus MR, et al. (2010) Hybrid odontogenic tumor with features of ameloblastic fibroodontoma, calcifying odontogenic cyst, and adenomatoid odontogenic tumor: a case report and review of the literature. $J$ Oral Maxillofac Surg 68: 470-474.

27. Wright JM, Kusama K (2017) Adenomatoid odontogenic tumor. In: World Health Organization Classification of Head and Neck Tumours. International Agency for Research on Cancer, Volume 9, Lyon, France, 221-222.

28. Hong SP, Ellis, GL, Hartman KS (1991) Calcifying odontogenic cyst: A review of ninety-two cases with reevaluation of their nature as cysts or neoplasms, the nature of ghost cells, and subclassification. Oral Surg Oral Med Oral Pathol 72: 56-64.

29. Gorlin RJ, Pindborg JJ, Clausen FP, et al. (1962) The calcifying odontogenic cyst: A possible analogue of the cutaneous calcifying epithelioma of Malherbe. Oral Surg 15: 1235-1243.

30. Carollo DA, Hoffman RL, Brodie AG (1971) Histology and Function of the Dental Gubernacular Cord. Angle Orthod 41: 300-307.

31. Praetorius F, Hjorting-Hansen E, Gorlin RJ, et al. (1975) Calcifying odontogenic cyst: Range, variations and neoplastic potential. Int J Oral Surg 4: 89.

32. Speight P, Ledesma-Montes C, Wright JM (2017) Calcifying odontogenic cyst. In: World Health Organization Classification of Head and Neck Tumours. International Agency for Research on Cancer, Volume 9, Lyon, France, 239-241.

33. Shear M (1994) Developmental odontogenic cysts: An update. J Oral Pathol Med 23: 1-11.

34. Fejerskov O, Krogh J (1972) The calcifying ghost cell odontogenic tumor or the calcifying odontogenic cyst. J Oral Pathol 1: 273287.

35. Ide F, Mishima K, Kikuchi K, et al. (2011) Development and growth of adenomatoid odontogenic tumor related to formation and eruption of teeth. Head Neck Pathol 5: 123-132.

36. Philipsen HP, Samman N, Ormiston IW, et al. (1992) Variants of the adenomatoid odontogenic tumor with a note on tumor origin. J Oral Pathol Med 21: 348-352.

37. Main DM (1970) The enlargement of epithelial jaw cysts: Odontol Revy 21: 29-49. 